\title{
Raising awareness: A year for multi-modality cardiac imaging
}

\author{
Eliana Reyes, MD, PhD, FESC ${ }^{\mathrm{a}, \mathrm{b}}$ \\ a King's College London and Guy's and St Thomas' NHS Foundation Trust PET Centre, London, \\ UK \\ b Nuclear Medicine Department, Royal Brompton and Harefield NHS Foundation Trust, London, \\ UK
}

Received Jun 4, 2018; accepted Jun 4, 2018

doi:10.1007/s12350-018-1335-3

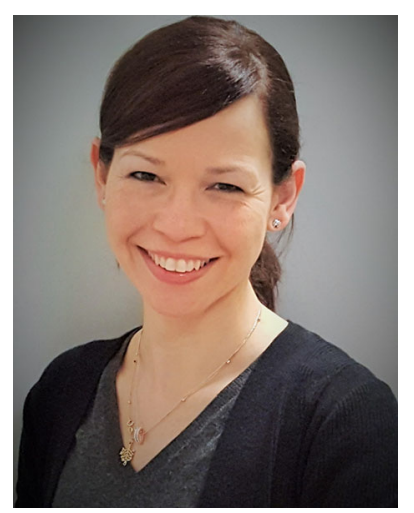

Eliana Reyes, Journal of Nuclear Cardiology Editorial Board and ASNC Publications Committee.

The year is still young, but 2018 has already been remarkable for cardiac imaging in the UK. For the first time, three cardiac imaging societies (the British Nuclear Cardiology Society, the British Society of Cardiovascular Magnetic Resonance, and the British Society of Cardiovascular Imaging/British Society of Cardiovascular CT) came together on May 2nd to hold the first national joint conference on nuclear cardiology, CMR, and cardiac CT imaging. The conference took place in the astonishing city of Edinburgh, which has been at the center of many political, cultural, and scientific contributions over centuries, therefore constituting an ideal backdrop for this landmark gathering. The meeting brought together experts from the three imaging modalities, who were joined by over 350 delegates. This event was more than the integration of the societies' annual meetings; it was a vivid example of what

\footnotetext{
Reprint requests: Eliana Reyes, MD, PhD, FESC, Nuclear Medicine Department, Royal Brompton and Harefield NHS Foundation Trust, Sydney Street, London, SW3 6NP, UK; e.reyes@rbht.nhs.uk J Nucl Cardiol 2018;25:1061-2. $1071-3581 / \$ 34.00$

Copyright (C) 2018 American Society of Nuclear Cardiology.
}

can be achieved with close collaboration and a shared goal of communicating scientific and clinical knowledge through an integrated, multi-modality imaging approach. As a cardiac imager, it was highly gratifying to be part of a much wider multi-talented community. Facilitated by a thoughtfully designed program, imaging-centered discussions moved fluidly from technique-specific indications to multi-modality applications for the investigation of heart disease. Imaging researchers and practitioners as well as trainees and allied professionals challenged each other openly in lively discussions about the current state of noninvasive cardiac imaging and its future.

The conference has been described by some as a huge success, reflecting the dedication and hard work of all the people involved in its organization and delivery. Indeed, its success was evidenced by its popularity and positive feedback. But there was another aspect of this remarkable achievement; the meeting succeeded in providing the right environment in which to raise awareness of a growing number of cardiac imaging enthusiasts with an interest in mastering the multimodality rather than the single imaging approach to the study of the cardiovascular system in health and disease.

According to the ADKAR model for change (Awareness, Desire, Knowledge, Ability, Reinforcement), ${ }^{1}$ awareness is the first of a series of steps to implement effective change; as in the ADKAR model, this national multi-modality imaging conference may represent the first link of a chain of events that may lead to a renewed, collaboration-centered practice of cardiac imaging in the UK.

As a proud and passionate nuclear cardiology practitioner, I welcome new initiatives and innovations in the field. Over recent years, we have witnessed a rapidly growing implementation of radiation dose reduction protocols; an increasingly number of studies looking at the feasibility and validity of CZT gamma-camera systems for quantitative myocardial perfusion; and, more recently, the 
use of deep learning to enhance the diagnostic performance of myocardial perfusion SPECT imaging. There is also fast-paced research on molecular imaging for the evaluation of atherosclerotic plaque vulnerability, and the assessment of infiltrative and inflammatory conditions of the heart. When looking at all these important advances, I cannot help but entertain the possibility of a future whereby innovation in nuclear cardiology, CMR, and Cardiac CT practices occur in an environment of partnership and close interdisciplinary collaboration between professionals from the three imaging disciplines. In such environment, the inability of one modality to deliver a particular application may be seen as an opportunity for the others, while their individual strengths are exploited to the full, aiming at complementing each other for the investigation of patients with cardiovascular disease.

While the "one-stop shop" remains an attractive concept in a world of increasingly limited resources, these may be better spent on optimizing and fine-tuning the capabilities of each of the imaging techniques. In the assessment of coronary artery disease, for example, the unique qualities of myocardial perfusion radiotracers, the tissue characterization properties of CMR, and the unmatched capabilities of CT for the visualization of the coronary arteries and characterization of atherosclerotic plaque should continue to be priority for scientific research and clinical validation. This does not imply curtailing the development of overlapping applications as long as they are complementing rather than competing alternatives. This approach may help minimize the duplication of effort, and the associated waste of time and resources that may incur when novel diagnostic applications develop in a parallel or overlapping manner rather than openly and interconnected. This may also help identify gaps in knowledge, and potentially new applications that, unlike established ones, would benefit greatly from the motivational incentive brought by competition.

In this regard, hybrid imaging may help deliver the “one-stop shop." Advanced PET/CT systems are now widespread, making it possible the simultaneous evaluation of myocardial perfusion and coronary anatomy, the detection and localization of cardiac inflammation and infection, and the characterization of atherosclerotic plaques. More recently, PET/MR systems become available and, although still largely employed for research purposes, cardiac PET/MR is likely to find an indication for clinical use in the not-too-distant future. ${ }^{2}$

The intertwining of radionuclide imaging with cardiac CT and CMR helps tilt the balance in favor of a multimodality, multi-disciplinary cardiac imaging practice. This brings the prospective of a multi-modality imaging professional. There will be no longer place for a nuclear cardiologist or a CMR specialist, but for a cardiac imager skilled in all three imaging modalities. But, should this be the case? Personal experience with hybrid PET/MR suggests that a far better setting is that where professionals from different clinical specialties with complementary expertise on nuclear cardiology, CMR, and cardiac CT join efforts to deliver a multi-modality imaging service while assisting medical physicists, biomedical engineers, computer programmers, and artificial intelligence (AI) specialists in the translation of benchside discoveries into bedside applications. Formal postgraduate training and the setting-up of a multi-modality imaging curriculum have long been discussed at national and international levels. Although this might be desirable, the increasing burden of information and fast-paced technological progress make it unrealistic for a single professional to master all imaging modalities at once. Instead, cardiac imagers may continue pursuing expertise in a single modality of their choice while gaining the necessary knowledge to appreciate the evolving role of each imaging technique in the diagnosis and management of heart disease. The multi-modality imaging professional may indeed become obsolete with the implementation of AI. Instead, training must prepare future imagers for the increasing adoption of artificially intelligent systems in research and clinical practices.

Whatever the strategy implemented to deliver state-ofthe-art, cost-effective cardiac imaging, this should transcend the interests of any single imaging group. Critical thinking toward evidence and current practices should be fostered so that the strengths and weaknesses of the several different imaging modalities are judged carefully and fairly. The first national multi-modality imaging conference in nuclear cardiology, CMR, and cardiac CT in the UK might have succeeded in raising awareness of a wider and powerful imaging community that shares a common purpose; it is now our mission to keep momentum and work toward an effective call to action to nurture interaction and bidirectional communication between imaging experts and allied disciplines so that improving patient-centered outcomes and making a more effective use of resources become the center of our coordinated efforts.

\section{Disclosure}

None declared.

\section{Reference}

1. King's College London. A guide to change management for managers and leaders. King's College London, London; 2017. pp. 1-23

2. Nensa F, Bamberg F, Rischpler C, Menezes L, Poeppel TD, la Fougère $\mathrm{C}$, et al. Hybrid cardiac imaging using PET/MRI: a joint position statement by the European Society of Cardiovascular Radiology (ESCR) and the European Association of Nuclear Medicine (EANM). Eur Radiol. 2018. https://doi.org/10.1007/s00330-017-5008-4 